\title{
Prevalencia e indicadores de riesgo de recesiones gingivales en adolescentes de Valdivia en el año 2018.
}

\section{Prevalence and risk indicators of gingival recessions in school senior students in the city of Valdivia in 2018.}

\author{
Yanina Amaro - Rivera ${ }^{1 *}$, Gabriela Alvarado -Negrón ${ }^{1}$, Nickol Manqui-Jara1
}

1. Instituto Odontoestomatología. Facultad de Medicina Universidad Austral de Chile.

* Correspondencia Autor: Yanina Amaro - Rivera | Dirección: Rudloff 1640, Valdivia, Región de los Ríos. Chiel. | Teléfono: +569 82489811 | E-mail: yamariv@gmail.com, yanina.amaro@uach.cl Trabajo recibido el 02/09/2019.

Aprobado para su publicación el13/02/2020

\section{RESUMEN}

Objetivo: Determinar la prevalencia de recesiones gingivales e identificar indicadores de riesgo, en estudiantes de cuarto medio de la ciudad de Valdivia en el año 2018. Material y métodos: Estudio de corte transversal. Se midió la prevalencia, distribución y tipo de recesión gingival según clasificación de Miller en estudiantes de cuarto medio de establecimientos públicos y subvencionados de la ciudad de Valdivia, seleccionados mediante muestreo aleatorio estratificado. Dos examinadores calibrados realizaron un examen clínico utilizando una sonda periodontal carolina del norte y un cuestionario escrito individual a cada estudiante para evaluar indicadores de riesgo. Los datos fueron tabulados y el análisis estadístico se realizó usando el programa estadístico SPSS 18 (IBM $®$ SPSS $®$ software). Resultados: Se examinaron 310 estudiantes. La prevalencia encontrada fue de $68,4 \%$. La Clase I de Miller se presentó en un $97,7 \%$. La arcada mandibular con un $64,8 \%$ presentó mayor prevalencia de recesiones gingivales y los premolares inferiores fueron los dientes más afectados con un $47,7 \%$. Conclusión: Existe una alta prevalencia de recesiones gingivales en la población estudiada. Los estudiantes de establecimientos públicos presentan significativamente mayor prevalencia de recesiones gingivales y menor frecuencia de cepillado.

PALABRAS CLAVE:

Prevalencia: Recesión gingival; Clasificación de Miller.

International Journal of Interdisciplinary Dentistry Vol. 13(1); 21-25, 2020.

\section{ABSTRACT}

Aim: To determine the prevalence of gingival recessions and identify risk indicators in school senior students in the city of Valdivia in 2018. Material and methods: Descriptive observational study. Prevalence, distribution and type of gingival recession were measured according to Miller classification in school senior students of public and charter establishments in the city of Valdivia, selected by stratified random sampling. A clinical examination was performed by two calibrated examiners using a North Carolina periodontal probe and an individual written questionnaire for each student to assess risk indicators. A descriptive analysis was performed using the statistical program SPSS 18 (IMB $^{\circledR}$ SPSS $^{\circledR}$ software). Results: 310 students were examined. The prevalence found of at least one gingival recession was $68.4 \%$. Miller's Class I was present in $97.7 \%$. The jaw presented a higher prevalence of gingival recessions with $64.8 \%$ and the lower premolars were the most affected teeth with $47.7 \%$. Conclusion: There is a high prevalence of gingival recessions in the population studied. Students in public establishments have a higher prevalence of recessions and a lower frequency of tooth brushing.

\section{KEY WORDS:}

Prevalence; Gingival Recession; Miller classification.

International Journal of Interdisciplinary Dentistry Vol. 13(1); 21-25, 2020.

\section{INTRODUCCIÓN}

La recesión gingival $(R G)$ es definida como el desplazamiento del margen gingival apical a la unión cemento-esmalte (LAC), con la exposición de la superficie radicular al ambiente oral. Es una condición antiestética, con posibles consecuencias como hipersensibilidad dental, caries y lesiones no cariosas, sangrado gingival y retención de placa bacteriana ${ }^{(1,2)}$. Al migrar la encía libre se genera una pérdida de encía adherida la cual si es severa puede causar dolor en función de masticación o durante el cepillado, provocando malestar al sujeto y favoreciendo la acumulación de placa bacteriana.

La detección de la RG en pacientes ha sido estudiada considerando distintos parámetros ${ }^{(3)}$, siendo la forma más sencilla y de rigor la medición de la distancia entre el margen gingival y el LAC estableciéndose como mínimo $1 \mathrm{~mm}$ de desplazamiento para considerarla como presente. Los principales factores etiológicos son la inflamación inducida por placa bacteriana y la abrasión mecánica. Se reconocen tres factores predisponentes principales que están asociados a una mayor susceptibilidad: biotipo gingival fino, condiciones mucogingivales y/o 
enfermedad periodontal inflamatoria(4). Diversos factores de riesgo para las RG han sido estudiados, a través de la aplicación de encuestas o cuestionarios sobre hábitos de higiene oral, técnica y frecuencia de cepillado, tipo de cepillo, uso de aparatología ortodóncica, tabaco, entre otros, $(5,6,7,8)$ pero aún existe controversia de la influencia individual de cada uno. Los estudios de prevalencia reportan diferentes magnitudes del problema, que va de $30 \%$ a $100 \%$, según la población estudiada y las edades de los sujetos $^{(9,10)}$. También se ha establecido que con la edad de la población existe un aumento en prevalencia, número de dientes afectados y severidad de las recesiones gingivales ${ }^{(4,8)}$.

Cuando no se asocia a enfermedad periodontal, los hábitos de higiene oral tienen un rol importante, sobre todo al considerar la frecuencia y la técnica de cepillado, el tipo de cepillo, junto con el uso de seda dental y/o colutorios en pacientes jóvenes ${ }^{(1)}$.

No existen datos en la ciudad de Valdivia de prevalencia de RG ni tampoco sobre los indicadores de riesgos asociados. El objetivo del presente trabajo fue determinar la prevalencia de RG en adolescentes de Valdivia, que cursaban cuarto medio durante el año 2018, e identificar los indicadores de riesgo presentes relacionados con los hábitos de higiene, exposición a tabaco y tratamientos de ortodoncia.

\section{MATERIAL Y MÉTODO}

Se realizó un estudio descriptivo de prevalencia de recesiones gingivales en una muestra representativa de estudiantes de cuarto medio de la ciudad de Valdivia. Junto con un examen clínico se incluyó la aplicación de un cuestionario de 11 preguntas elaborado para la investigación, con el objetivo de determinar la presencia de indicadores de riesgo. Previo a su aplicación se realizó una prueba piloto del cuestionario a 41 estudiantes voluntarios del primer semestre del año 2018, de la carrera de odontología de Universidad Austral de Chile. Se evalúo la apariencia y factibilidad de aplicación del instrumento. No se realizaron otras pruebas de validación.

Población: La población de estudio fueron jóvenes estudiantes que cursan cuarto año medio en la ciudad de Valdivia de todos los establecimientos educacionales de régimen diurno, de tipo público, privado y subvencionado. Debido a la negativa de participar de los establecimientos privados de la ciudad $(10,4 \%$ de la matricula total). El tamaño de la muestra se determinó con la matricula proporcionada por el MINEDUC correspondiente a los establecimientos públicos y subvencionados. La muestra se constituyó por aquellos estudiantes que se encontraron presentes el día de la revisión en cada establecimiento. Criterios Inclusión: Alumnos que presentaron el consentimiento informado firmado, ya sea por sus padres o tutores en el caso de menores de edad y/o por el propio participante mayor de edad. Criterios Exclusión: Presencia de enfermedad periodontal destructiva, tratamiento periodontal activo, patología oral aguda que dificulte examen clínico y/o menos de veinte dientes permanentes presentes en boca.

Control de fuentes de Sesgos: Se realizó un muestreo estratificado por tipo de establecimiento educacional y un muestreo aleatorio simple con el listado de alumnos matriculados para la selección de éstos.

Previo a la recolección de los datos los observadores realizaron un proceso de calibración que consistió en la observación y clasificación de una serie de imágenes de recesiones gingivales según la clasificación de Miller ${ }^{(12)}$, los que previamente fueron diagnosticadas por un especialista en periodoncia. Se calculó el grado de acuerdo mediante el test de Kappa el cual dio un valor de $0.84(p=0,00)$. Durante el proceso de examen clínico, frente a una duda ambos observadores llegaron a acuerdo mediante consenso. Los datos obtenidos se registraron en una ficha clínica, para luego ser ingresados por ambos investigadores en una planilla digital.

Cálculo tamaño muestral: Para el cálculo del tamaño de muestra se utilizó el programa Epidat (versión 4.2, julio 2016). Se consideró una prevalencia estimada (variabilidad) del $60 \%{ }^{(5)}$. La matrícula de estudiantes de establecimientos públicos y subvencionados cursando cuarto año medio el año 2018 (según datos de la Secretaría Ministerial de Educación) fue de 1670. Con un intervalo de confianza del 95\%, una potencia del $80 \%$ y un margen de error esperado del $5 \%$, se obtuvo un tamaño muestral de 303 alumnos. Para el muestreo estratificado por tipo de establecimiento educacional, se consideró el porcentaje de matrícula con respecto al total de estudiantes. Para seleccionar los establecimientos, se realizó un muestreo aleatorio simple. Si el establecimiento rechazaba la inclusión en el estudio se repetía el proceso de aleatorización con otro. Aspectos éticos: El protocolo del estudio fue aprobado por parte del Comité de Ética Científico del Servicio de Salud de Valdivia y revisado por el Departamento de Educación Municipal (DAEM) de la llustre Municipalidad de Valdivia.

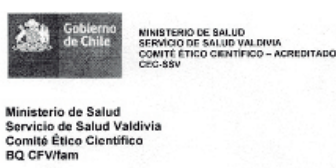

ORD.: 142

ANT.: Ord. 058 del 13.03 .2018

MAT.: Informa lo que indica

Valdivia, 24.04.2018

DE: BQ. Carlos Fernàndez Veg Presidente Comité Etico Científico Servicio de Salud Valdivia

A: Dra. Yanina Amaro Rivera Patrocinante de Tesis Escuela de Odontologia UACH

En relación a documento del antecedente, donde se envian los requerimiento para aprobación del Proyecto de Investigación titulado: "Prevalencia de rocesiones para aprobación del Proyecto de Investigación titulado: 'Prevalencia de rocesiones
gingivales en adojescentes de Valdivia en el año 2018', informo a usted, que este Comité considero lo siguiente:

El proyecto y documentación revisada NO PRESENTA repararos éticos, metodológicos, legales. Sólo podrán participar de este protocolo los participantes que sean capaces de Consentir.

El diseño se ajusta a las normas de Investigación en Seres Humanos.

La razón de riesgo/beneficio fue estimada favorable para el participante.

El formulario de consentimiento informado cumple con los requisitos exigidos.

Además de conocer los antecedentes presentados, el Comité revisó y aprobó los siguientes documentos:

1. Documento de Información para el Participante y Formulario de Asentimiento Informado.

2. Documento de Consentimiento Informado Parental.

En consecuencia, el CEC-SSV AUTORIZA el estudio presentado.

El Investigador se compromete a respetar la legislación vigente, normas técnicas y recomendaciones nacionales e internacionales sobre investigacion cientifica los registros clinicos y de investigación en seres humanos, de acuerdo con la Ley $N^{*} 19628$, Ley $N^{*} 20120$, Ley $N^{\circ} 20584$, sus reglamentos de aplicación y modificaciones.

El investigador se compromete a respetar el Reglamento Interno del CEC-SSV y a utilizar sólo la documentación que fue aprobada y autorizada por este CEC, las cuales se entregan firmadas y timbradas para ser copiadas desde la versión original autorizada.

Los datos utilizados y la información obtenida se limitan a lo expresado en la formulación del proyecto mencionado. No se autoriza otro uso. Esta aprobación tiene vigencia de un año, a contar de la fecha de emisión del presente oficio.

Es responsabilidad del Investigador tramitar la autorización correspondiente con el Director(a) de la Institución en la cual se ejecutará el proyecto y en las instituciones que sea pertinente hacerlo y enviar un informe de avance o informe final segủn corresponda, a este CEC con plazo máximo Abril/2019, por escrito y via oficina de partes del SSV, tanto para solicitar prosecución del estudio, re-aprobación anual, asi como también para requerir toma de conocimiento en caso de finalización yfo cierre del Centro o del Estudio.

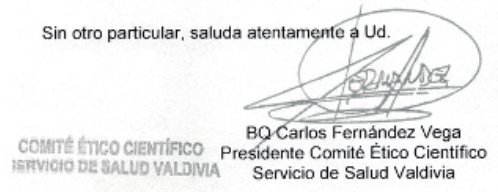

Anexo 1: Comprobante Comité de Etica Servicio de Salud de Valdivia

Recolección de datos: Los participantes fueron incluidos en forma voluntaria, sólo se evaluaron los estudiantes que firmaron el consentimiento informado o por su apoderado en el caso de ser menor de edad. Durante el examen intraoral el participante estuvo sentado en una silla y el examinador de pie, utilizando una linterna frontal, guantes y mascarillas. Se procedió a identificar las RG después de la aplicación de un Examen Periodontal Básico, realizado con sonda periodontal OMS (Hu Friedy) para descartar presencia de enfermedad periodontal destructiva. Se consideró presencia de RG cuando se observó exposición de la raíz por cara vestibular de cualquier diente presente en boca, cuyo desplazamiento del margen gingival desde el LAC sea $>1 \mathrm{~mm}$. Para medir el desplazamiento del margen gingival se utilizó una sonda periodontal Carolina Del Norte (Hu Friedy, Alemán).

Luego se determinó la presencia de indicadores de riesgo aplicando un cuestionario de forma escrita, auto llenado y con los examinadores presentes en caso de consultas. Al finalizar se realizó instrucción de higiene a cada participante. Los datos fueron recolectados durante los meses de agosto y septiembre del 2018.

Análisis Estadístico : Se realizó un análisis descriptivo e inferencial con el software SPSS para Windows ${ }^{\circledR} 19$, SPSS Inc., Chicago, III. USA. Se consideró como nivel mínimo de significancia estadística un valor de $\mathrm{p}<0.05$. 


\section{RESULTADOS}

La muestra total estuvo formada por 310 adolescentes, de ellos el $53,5 \%$ fueron mujeres y el $46,2 \%$ hombres pertenecientes a 6 establecimientos públicos y 5 subvencionados de la ciudad de Valdivia. Las características de la muestra por sexo y tipo de establecimiento se muestran en la tabla 1.

Tabla 1: Características de la muestra según sexo y tipo de establecimiento

\begin{tabular}{lcc|lcc|cc}
\hline \multicolumn{2}{c|}{ Sexo } & \multicolumn{3}{|c|}{ Establecimiento } & \multicolumn{2}{c}{ Edad } \\
& $\mathbf{n}$ & $\%$ & & $\mathbf{n}$ & $\%$ & $\overline{\mathbf{x}}$ & DS \\
\hline Masculino & 139 & 44,8 & Público & 215 & 69,4 & 17,6 & $\pm 0,74$ \\
Femenino & 171 & 55,2 & Subvencionado & 95 & 30,6 & Rango & $16-21$ \\
\hline Total & 310 & 100 & & 310 & 100 & &
\end{tabular}

El $68,4 \%(I C=63,1 \%-73,4 \%)$ de los participantes presentaron al menos una RG, con un total de 907 dientes afectados y un promedio de 2,9 $\pm 3,49$ recesiones. No se observó diferencia estadísticamente significativa entre géneros, con una prevalencia de $70,5 \%$ (IC $=63,6 \%$ $78,8 \%$ ) en hombres y $66,7 \%$ ( IC $=58,9 \%-73,2 \%$ ) en mujeres. Al analizar la prevalencia y promedio de RG por establecimiento, se encontró diferencia estadísticamente significativa $(p=0,03)$ entre los dos tipos de establecimientos. Los colegios públicos alcanzaron una prevalencia de $75,3 \%$ con un promedio de 3,6 RG por estudiante versus una prevalencia del $52,2 \%$ y un promedio de 1,4 RG por estudiante de los establecimientos subvencionados.

Los valores de prevalencia y promedio de RG según tipo de establecimiento y género se detallan en tabla 2.

La Clase I de Miller fue el tipo de RG más frecuente en un $97,7 \%$, seguida por la clase II con una frecuencia de $1,4 \%$, y Clase III con una frecuencia de $0,9 \%$

La distribución de la RG, se analizó según el tipo de diente afectado y arcada en que se encontraba, el tipo de diente más afectado fueron los premolares mandibulares con una frecuencia del $47,4 \%$, seguido por los caninos mandibulares. La mandíbula con un $64,8 \%$ fue la arcada con mayor prevalencia de RG, datos se muestran en la tabla 3

Tabla 3: Prevalencia de RG por tipo de diente y arcada.

\begin{tabular}{ccccccc}
\hline & \multicolumn{5}{c}{ Frecuencia de dientes afectados por RG (\%) } \\
\cline { 2 - 6 } & Incisivos & Caninos & Premolares & Molares & Arcada \\
\cline { 2 - 6 } Maxilar & 17 & 43 & 65 & 36 & 105 \\
& $(5,5 \%)$ & $(13,5 \%)$ & $(21 \%)$ & $(11,6 \%)$ & $(33,9 \%)$ \\
Mandíbula & 68 & 81 & 147 & 44 & 201 \\
& $(21,9 \%)$ & $(26,1 \%)$ & $(47,4 \%)$ & $(14,2 \%)$ & $(64,8 \%)$
\end{tabular}

De los resultados de la encuesta se identificó, en relación a los indicadores de riesgo, que un $98,6 \%$ de la muestra se cepillaba los dientes, existiendo una diferencia estadísticamente significativa en relación a prevalencia de $R G(p=0,01)$ entre los estudiantes que se cepillaban 2 o 3 veces al día versus los que lo realizaban 1 vez al día; y entre el número de RG por estudiante en relación a los que se cepillaban 3 veces al día en comparación a los que no lo realizaban. El 30,3\% de los estudiantes realizaban higiene interproximal y un $40,3 \%$ desconocían el tipo de cerdas de su cepillo de dientes. Respecto al consumo de tabaco un total de $31,9 \%$ de estudiantes afirmó fumar cigarrillo.

Tabla 2: Prevalencia y promedio de RG por tipo de establecimiento y sexo.
El resumen de las frecuencias por respuesta y sus respectivos valores de prevalencia y promedio de RG por cada ítem de la encuesta se muestran en la tabla 4.

El $34,7 \%$ de los estudiantes de establecimientos subvencionados declaró cepillarse más de dos veces al día versus un $22,3 \%$ de los estudiantes de establecimientos públicos $(p=0,02)$. Entre los estudiantes de establecimientos públicos hubo mayor proporción de quienes se cepillan una vez o menos al día 13,9\%, comparados con los de establecimientos subvencionados que fueron $7,3 \%$. Esta diferencia no alcanzó significancia estadística. La mayor prevalencia de RG fue entre quienes se cepillan los dientes una vez o menos al día en ambos tipos de establecimientos. Los estudiantes de establecimientos públicos que se cepillan los dientes 2 veces al día tuvieron mayor prevalencia de RG que sus pares de establecimientos subvencionados con igual frecuencia de cepillado, 76,6 versus 47,2 ( $p=0,00$ ).

Los datos de frecuencia de cepillado y prevalencia de RG por tipo de establecimiento se presentan en tabla 5.

\section{DISCUSIÓN}

El presente estudio arrojó una prevalencia de RG estimada de $68,4 \%$ (IC $=63,1 \%-73,4 \%)$. Se estima que más del $60 \%$ de la población humana tiene al menos una recesión gingival(14). Dos estudios realizados en Chile, uno por Beltrán ${ }^{(11)}$ en Temuco observó una prevalencia del $82,2 \%$ de recesiones gingivales en un rango de edad entre 18-34 años, mayor a lo reportado por Wilckens. ${ }^{(15)}$ que evaluó a estudiantes de odontología en la Universidad de Concepción y determinó una prevalencia del $67,5 \%$; similar a lo encontrado en el presente estudio. La diferencia en la prevalencia con el estudio de Temuco se puede atribuir al mayor rango de edad y a la no exclusión de sujetos con periodontitis. En poblaciones donde no hay exclusión de individuos con periodontitis se reportan prevalencias mayores que la encontrada en el presente estudio ${ }^{(13,8)}$.

Los valores de prevalencia y promedio de RG por sujeto, fueron mayores en los establecimientos públicos que en los subvencionados. En Chile el sistema educativo se caracteriza por ser altamente segregado por condición socioeconómica, los estudiantes de colegio públicos tienen mayor nivel de vulnerabilidad económica que estudiantes de colegios subvencionados o particulares pagados. La no participación de los establecimientos particulares en el presente estudio limitó la posibilidad de poder comparar esta variable de forma completa y asumir el supuesto que la condición socioeconómica de los estudiantes puede influir en aumentar la prevalencia de RG, actuando como un determinante social que influye en esta condición, tal como se observa en otras patologías prevalentes en salud oral. A pesar que las RG son una condición ampliamente prevalente en la población a nivel mundial, y que la literatura no ha reportado que la condición socioeconómica influya en aumentar la prevalencia, es posible que los estudiantes de colegios públicos pertenezcan a una población con menor acceso a instrucción en medidas de higiene oral eficiente y atraumático y/o a atención profesional oportuna que permita corregir factores anatómicos desfavorables como malposiciones dentarias u otros que favorezcan la formación de RG. Es necesario en futuras investigaciones analizar estas dimensiones para poder identificar cuáles son los factores que puedan explicar integralmente estos hallazgos.

De los resultados obtenidos en el cuestionario se determinó asociación significativa entre baja frecuencia de cepillado y prevalencia y número de RG por persona.

La presencia de RG en un diente es el resultado final de múltiples factores que interactúan, tanto morfológicos como funcionales. Una pobre higiene oral ha sido señalado como un factor funcional externo que promueve un estado inflamatorio que favorece la formación de la $\mathrm{RG}^{(10)}$. Merijohn identifica como causas primarias de recesión gingival en sujetos susceptibles la inflamación provocada por placa bacteriana

Tipo de Establecimiento

\begin{tabular}{|c|c|c|c|c|c|c|c|c|c|c|c|c|c|}
\hline & \multicolumn{5}{|c|}{ Público } & \multicolumn{4}{|c|}{ Subvencionado } & \multicolumn{4}{|c|}{ Total } \\
\hline & \multicolumn{3}{|c|}{ RG + } & \multicolumn{2}{|c|}{ Cantidad } & \multicolumn{2}{|c|}{ RG + } & \multicolumn{2}{|c|}{ Cantidad } & \multicolumn{2}{|c|}{ RG + } & \multicolumn{2}{|c|}{ Cantidad } \\
\hline & $\mathrm{n}$ & $\%$ & $\bar{x}$ & DS & $\mathrm{n}$ & $\%$ & $\overline{\mathrm{x}}$ & DS & $\mathrm{n}$ & $\%$ & $\bar{x}$ & DS & \\
\hline \multirow{2}{*}{ Sexo } & $M$ & 79 & 76,7 & 4,0 & $\pm 4,31$ & 19 & 52,8 & 1,3 & $\pm 1,59$ & 98 & 70,5 & 3,3 & $\pm 3,98$ \\
\hline & $\mathrm{F}$ & 83 & 74,1 & 3,2 & $\pm 3,31$ & 31 & 52,5 & 1,5 & $\pm 1,95$ & 114 & 66,7 & 2,6 & $\pm 3,03$ \\
\hline Total & 162 & 75,3 * & $3,6^{*}$ & $\pm 3,83$ & 50 & $52,6^{*}$ & $1,4^{*}$ & $\pm 1,82$ & 212 & 68,4 & 2,9 & $\pm 3,49$ & \\
\hline
\end{tabular}

* Indica resultado con significancia estadística $(p<0.05)$ 
Tabla 4: Resultados cuestionario de Hábitos de higiene, ortodoncia y tabaco.

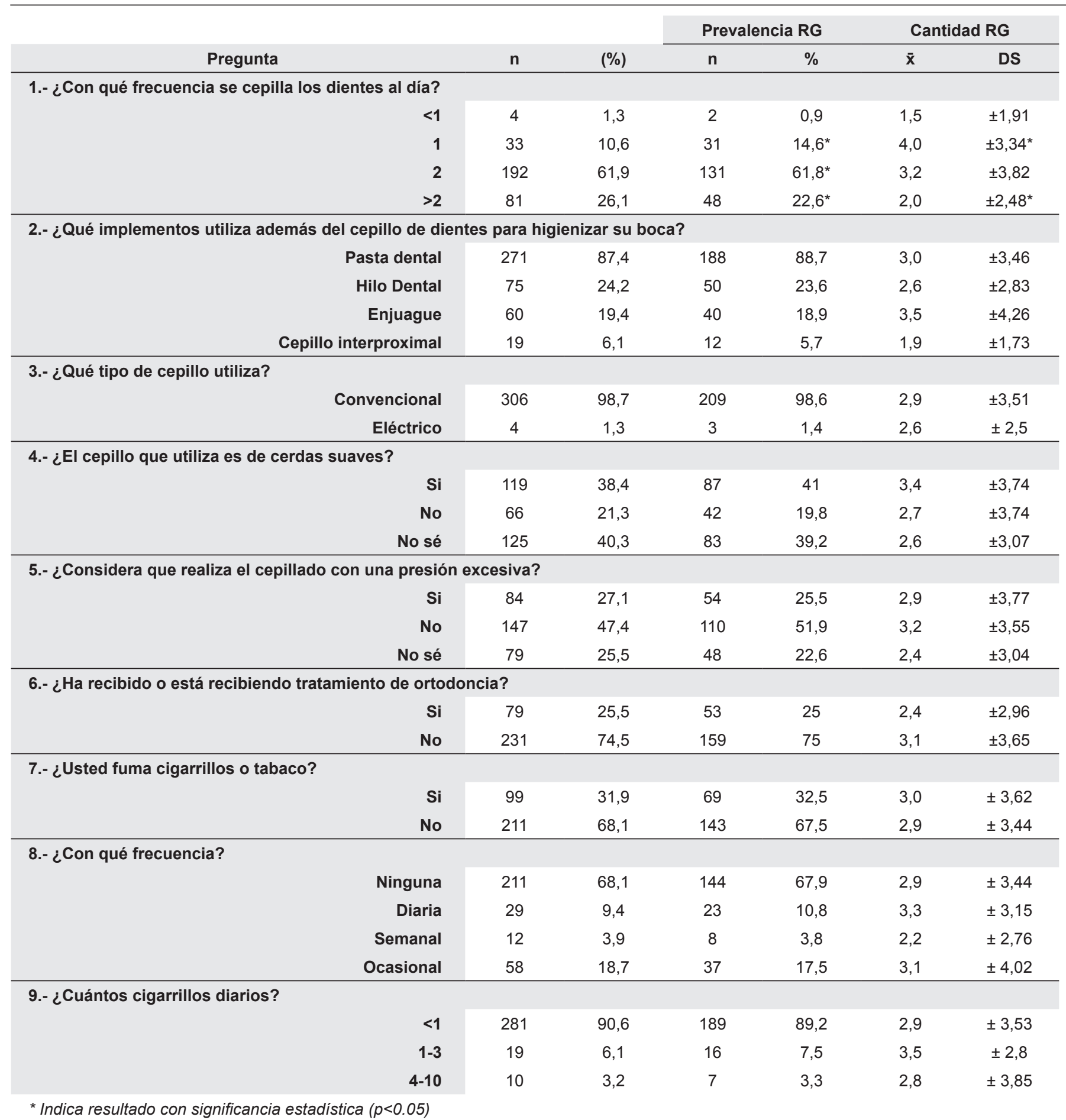

Tabla 5: Prevalencia de Recesiones Gingivales según frecuencia de cepillado por tipo de establecimiento.

\begin{tabular}{|c|c|c|c|c|c|c|c|c|c|c|c|c|}
\hline \multirow{3}{*}{ Frecuencia de Cepillado } & \multicolumn{4}{|c|}{ Públicos } & \multicolumn{4}{|c|}{ Subvencionados } & \multicolumn{4}{|c|}{ Total } \\
\hline & \multirow[b]{2}{*}{$\mathrm{n}$} & \multicolumn{3}{|c|}{$R G+$} & \multirow[b]{2}{*}{$\mathrm{n}$} & \multicolumn{3}{|c|}{$R G+$} & \multirow[b]{2}{*}{$\mathrm{n}$} & \multirow[b]{2}{*}{$(\%)$} & \multicolumn{2}{|c|}{$R G+$} \\
\hline & & $\%$ & $n$ & $\%$ & & $\%$ & $\mathrm{n}$ & $\%$ & & & $n$ & $\%$ \\
\hline 1 al día o menos & 30 & 13,9 & 26 & 86,6 & 7 & 7,3 & 7 & 100 & 37 & 11,9 & 33 & 89,18 \\
\hline 2 al día & 137 & 63,7 & 105 & 76,6 * & 55 & 57,8 & 26 & 47,2 * & 192 & 61,9 & 131 & 68,22 \\
\hline$>2$ al día & 48 & 22,3 * & 31 & 64,5 & 33 & $34,7^{*}$ & 17 & 51,5 & 81 & 26,1 & 48 & 59,25 \\
\hline Total & 215 & & 162 & 75,3 & 95 & & 50 & 52,6 & 310 & & 212 & 68,3 \\
\hline
\end{tabular}


y la acción mecánica de un cepillado abrasivo(4). La mayor prevalencia de RG y mayor número de RG por persona entre quienes se cepillan 1 vez o menos al día, se puede atribuir a un insuficiente control de placa bacteriana con presencia de inflamación gingival en esta población.

Beltrán encontró relación significativa entre mayor frecuencia de cepillado y prevalencia de RG, a diferencia de los resultados del presente estudio. Sin embargo también reportaron que el sangrado gingival, se relacionó con mayor frecuencia de $\mathrm{RG}^{(16)}$. Los antecedentes señalados demuestran que es necesario evaluar tanto frecuencia, tipo y eficiencia del cepillado, pues el factor inflamatorio se observa como relevante en la formación de RG en población joven, y es factible mediante educación e instrucción poder intervenir este aspecto.

Hubo mayor afección de la arcada mandibular en concordancia con otros estudios ${ }^{(12,5,6,8)}$.Matas..$^{(7)}$ en Barcelona realizó un estudio longitudinal prospectivo con un promedio de 23,5 años de edad, determinando al inicio del estudio que los caninos eran los dientes más afectados, pero 10 años después los resultados demostraron mayor afección de molares, sin diferencias por arcada.

El uso de ortodoncia puede ser un factor predisponente de RG según la literatura ${ }^{(10,5,1)}$ el $25,5 \%$ de los individuos tenía historia de tratamiento de ortodoncia y no hubo relación con la prevalencia de RG ni con el promedio de RG por individuo. Tampoco se encontró asociación con el consumo de cigarrillo, a pesar de que el $31,9 \%$ de la muestra eran fumadores. Estos resultados concuerdan con Matas que tampoco encontró relación respecto al uso de ortodoncia y tabaco con $R^{(7)}$.

Al estudiar qué elementos de higiene se utilizaban en forma adicional al cepillo, un $24,2 \%$ de la muestra señaló usar hilo dental. El $40,3 \%$ desconoce la dureza de las cerdas del cepillo que utiliza. Se concluye que en la población estudiada no existe un adecuado conocimiento sobre las características deseables de los elementos de higiene oral y existe una baja frecuencia de higiene interproximal

De acuerdo con los resultados es posible concluir que existe en esta población una relación entre baja frecuencia de cepillado y mayor prevalencia de recesiones gingivales. Entre los jóvenes que presentan misma frecuencia de cepillado (2 veces al día), los que pertenecen a establecimientos públicos tienen mayor prevalencia de RG.

\section{RELEVANCIA CLÍNICA}

Este estudio aporta información epidemiológica nueva sobre prevalencia y posibles indicadores de riesgo de recesiones gingivales en la población escolar de cuarto año medio de la ciudad de Valdivia. Entregando información sobre uso de elementos de higiene, frecuencia de cepillado y prevalencia de tabaquismo. Esta información es valiosa para reforzar estrategias de prevención y educación en esta población.

\section{DECLARACIÓN DE CONFLICTOS DE INTERÉS}

Los autores declaran no tener conflictos de interés ni fuentes de financiamiento externos.

\section{Bibliografía}

1. García R, Bujaldón A, Rodríguez A. Parámetros clínicos y periodontales predictores de la severidad de la recesión gingival. Gac Med Mex. 2016;152:51-58. 2. Pradeep K, Rajababu P, Satyanarayana D, Sagar V. gingival recession: review and strategies in treatment of recession. Case Rep Dent. 2012;2012:563421.

3. Bhusari P, Agrawal N, Upadhyay S, Verma S, Jain A, Jaroli S. Classification \& prevalence of dental surface defects in areas of gingival recession -a clinical study. J Clin Diagn Res. 2014;8(7):1-4.

4. Merijohn GK. Management and prevention of gingival recession. Periodonto 2000. 2016:71(1):228-42

5. Chambrone L, Bonazzio C, Chambrone LA. Traumatic gingival recession in denta students: Prevalence, severity and relationship to oral hygiene. Can J Dent Hygiene. 2013;47:(2):78-82

6. Chrysanthakopoulos NA. Prevalence and associated factors of gingival recession in Greek adults. J Investig Clin Dent. 2013;4(3):178-85.

7. Matas F, Sentís J, Mendieta C. Ten-year longitudinal study of gingival recession in dentists. J Clin Periodontol. 2011;38:1091-98.

8. Seong J, Bartlett D, Newcombe R, Claydon N, Hellin N, West NX. Prevalence of gingival recession and study of associated related factors in young UK adults. J Dent. 2018;76:58-67.
9. Teixeira D, Zeola L, Machado A, Gomes R, Souza P, Mendes D. Relationship between non carious cervical lesions, cervical dentin hypersensitivity, gingival recession, and associated risk factors: A cross-sectional study. J Dent. 2018;76: 93-97.

10. Dominiak M, Gedrange T. New perspectives in the diagnostic of gingival recession. Adv Clin Exp Med. 2014;23(6):857-63

11. Beltrán V, Silva M, Padilla M, Aillapan E, Sanhueza A, Cantín M. Morphological patterns of gingival recession in adult chilean population. Int $\mathrm{J}$ Morphol. 2013;31:1365-70.

12. Miller PD. A classification of marginal tissue recession. Int $\mathrm{J}$ Periodontics Restorative Dent. 1985;5:8-13

13. Chang D. Comparison of Age and Sex Regarding Gingival and Papillary Recession. Intern J Periodontics Restorative Dent. 2012;32(5):555-61.

14. Allen R. Treating gingival recession. Irish Dentist. 2011 July:20-26

15. Wilckens M, Merino J, Rios M. Recesión gingival y cepillado en estudiantes de odontología. Periodoncia. 2003;13(4):281-88

16. Beltrán V, De la Rosa G, Wilckens M, Fuentes R, Padilla M, Aillapan E. Effects of manual toothbrushing on gingival recession in an adult population sample in south of Chile. Int J Odontostomat. 2014;8(3):461-67. 